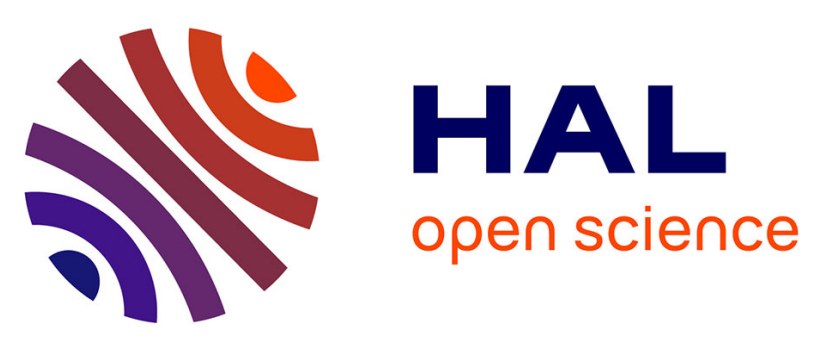

\title{
Joint state and parameter estimation for a class of cascade systems: Application to a hemodynamic model
}

Chadia Zayane-Aissa, Da-Yan Liu, Taous-Meriem Laleg-Kirati

\section{To cite this version:}

Chadia Zayane-Aissa, Da-Yan Liu, Taous-Meriem Laleg-Kirati. Joint state and parameter estimation for a class of cascade systems: Application to a hemodynamic model. The 13th European Control Conference (ECC 2014), Jun 2014, Strasbourg, France. hal-00987907

\section{HAL Id: hal-00987907 https://hal.inria.fr/hal-00987907}

Submitted on 6 May 2014

HAL is a multi-disciplinary open access archive for the deposit and dissemination of scientific research documents, whether they are published or not. The documents may come from teaching and research institutions in France or abroad, or from public or private research centers.
L'archive ouverte pluridisciplinaire HAL, est destinée au dépôt et à la diffusion de documents scientifiques de niveau recherche, publiés ou non, émanant des établissements d'enseignement et de recherche français ou étrangers, des laboratoires publics ou privés. 


\title{
Joint state and parameter estimation for a class of cascade systems: Application to a hemodynamic model
}

\author{
Chadia Zayane-Aissa, Da-Yan Liu and Taous-Meriem Laleg-Kirati
}

\begin{abstract}
In this paper, we address a special case of state and parameter estimation, where the system can be put on a cascade form allowing to estimate the state components and the set of unknown parameters separately. Inspired by the nonlinear Balloon hemodynamic model for functional Magnetic Resonance Imaging problem, we propose a hierarchical approach. The system is divided into two subsystems in cascade. The state and input are first estimated from a noisy measured signal using an adaptive observer. The obtained input is then used to estimate the parameters of a linear system using the modulating functions method. Some numerical results are presented to illustrate the efficiency of the proposed method.
\end{abstract}

Key Words: Joint state and parameter estimation, Adaptive observers, Modulating functions method, Balloon Model, fMRI

\section{INTRODUCTION}

Joint state and parameter estimation is crucial for the study of physical systems. It is generally oriented either to the identification or to the design of control laws for the studied systems. This problem has been the field of intensive research especially for nonlinear systems, and the proposed methods are generally built using recursive algorithms. An intuitive approach consists in including the parameters in the state vector and estimating the augmented system using observerbased approaches. The most widespread approach falling in this category is the Extended Kalman Filter (EKF), which is considered as first order approximations of nonlinear systems. Nonetheless, it may result in biased estimations or divergence [16]. Some systems structures, however, allow to benefit from advanced techniques in both areas of nonlinear state estimation and parameter estimation. The scope of this work is restricted to state and parameter estimation problems for systems that have directly or can be derived into such structures. Among the techniques commonly used for nonlinear state estimation, some are based on change of state variables and output injection. They try to handle the nonlinearities via state feedback by expressing them as a function of inputs and outputs, mainly using Lie differentiation [12]. The output injection uses robust observers, such as high gain and/or sliding mode observers. These techniques are generally sought to estimate hidden state and inputs [21]. The effectiveness of these methods is subject to the existence

C. Zayane-Aissa and T.M. Laleg-Kirati are with Computer, Electrical and Mathematical Sciences and Engineering Division, King Abdullah university of science and technology (KAUST), Kingdom of Saudi Arabia, \{chadia.zayane, taousmeriem. laleg\}ekaust. edu.sa.

Da-Yan Liu is with INSA Centre Val de Loire, Université d'Orléans, PRISME EA 4229, Bourges Cedex 18020, France, dayan.liudinsa-cvl.fr. of such a transformation and its computational ease, which is not the case for a wide class of physical systems, especially in neuroscience. Moreover, they may require satisfying some rank conditions that can hardly be satisfied especially for the class of single output systems. Recently, other techniques, such as Linear Matrix Inequalities (LMI) [9], have been suggested for some specific cases. But, their convergence relies upon heavy assumptions.

As for parameter identification, two main approaches have been considered: direct approaches and indirect approaches [22]. For direct identification approaches, there are two main issues: the first one is concerned with the estimation of the derivatives of the input-output signals, and the second one is due to the measurement noise [8]. Modulating functions method is one of the major direct identification approaches for continuous-time systems. This method was proposed by Shinbrot in 1957 [24]. The idea was inspired by Laplace and Fourier transforms. Generally, the use of modulating functions allows to transform the differential equation of analyzed noisy signals, into a sequence of algebraic integral equations. Then, parameters can be estimated by solving a linear system. Unlike the classical parameter estimation techniques such as the least square and the gradient methods, the modulating function technique presents the advantage of avoiding the computation of noisy output signals derivatives. Moreover, this method takes advantage from the low-pass filtering property of integrals [5], [15]. It has been successfully used for parameter identification for nonlinear systems, time-varying systems, fractional order systems and noisy sinusoidal signals [19], [14], [15].

In this work, we take advantage of both nonlinear state and input estimation techniques and parameter identification methods to estimate some key variables that allow the understanding of a brain region behavior using functional Magnetic Resonance Imaging (fMRI).

fMRI experiments consist in scanning the brain while performing a specific task. The collected data, called Blood Oxygenation Level Dependent (BOLD), is an indirect measurement of the brain activity, and allows the mapping between the neural activity and the active brain region. Among the hemodynamic models used to relate the changes in the blood flow, volume and oxygenation level to the neural activity in a localized area of the brain, the Balloon model has been first proposed by Buxton et. al. [3]. Other variants of the Balloon model have been developed, such as Friston's variant [6] which is used in this paper. The main feature of the Balloon model is that it describes the BOLD signal as a nonlinear combination of the blood 
volume and deoxyhemoglobin content, which are expressed as nonlinear functions of the neural activity. Despite the efforts that have been made to construct reliable models describing all the phenomena involved in the chain from the neural activity to the BOLD response, there is still a great challenge in the joint estimation of the parameters and the non measured states. The first important attempt to characterize the hemodynamic response has been proposed by Friston et. al. using Volterra Kernel expansion [6]. Riera et. al. [23] proposed to use a local linearization filter to identify the parameters of Friston's model [6] taking into account the noise. The local linearization can be seen as a form of extended Kalman filter. However, this estimation had to be contained in a specific vector space, otherwise the problem was underdetermined and the estimation of the input has been performed with a restrictive parametrization. Deneux et. al. [4] used a Maximum Likelihood approach to estimate the parameters of different versions of the balloon model. They also studied the identifiability of the parameters. In [7], a variational Bayesian technique, called Dynamic Expectation Maximization, has been proposed to perform a complete deconvolution of fMRI data enabling the estimation of the parameters, the sates and the input. Havlicek et. al. [10] proposed a nonlinear Kalman filtering based on an efficient square-root cubature Kalman filter for the inversion of nonlinear stochastic dynamic causal models. However, this approach leaves some limitations.

This paper is organized as follows. In Section II, we present a wide class of cascade systems, under which falls the Balloon model. Section III is dedicated to the application of the adaptive observers and the modulating functions method to the hemodynamic Balloon model. In Section IV, we show some numerical results to illustrate the performance of the proposed method.

\section{PROBLEM STATEMENT}

In this section, we present the proposed approach to estimate both the state components and some parameters for a special class of cascade systems.

The estimation of state and parameters for nonlinear systems has been addressed in the general framework of adaptive observers for nonlinear systems. To the best of our knowledge, the problem has been widely studied for the class of state affine systems of the form:

$$
\left\{\begin{array}{l}
\dot{x}=\phi(x, u, t)+\psi(x, u, t) \theta \\
y=h(x)
\end{array}\right.
$$

where $x$ denotes the hidden state, $u$ is the known input, $\theta$ is a set of unknown parameters, $\phi$ and $\psi$ are nonlinear functions. For systems of form (1), adaptive observers, initially proposed by [1] in the nonlinear case, are commonly applied to estimate the unknowns. Basically, it can be viewed as an observer where the parameters in $\theta$ are constant, a dynamic equation for $\theta: \dot{\hat{\theta}}=F(\hat{x}, y, u, t)$ is added such that, under some persistently exciting condition of $u, \hat{\theta}$ converges to $\theta$. For this purpose, an unified approach consists in transforming the system into some canonical form, also called adaptive observer form [18], [17]. After the change of coordinates, the system (1) can be written as follows:

$$
\left\{\begin{array}{l}
\dot{\zeta}=A \zeta+\alpha(y, u)+B \beta(y, u, t) \theta \\
y=C \zeta
\end{array}\right.
$$

where now $(A, C)$ is in the Brunovsky canonical form, and $B$ is a vector of appropriate size. Note that there exists another adaptive observer form similar to (2), for a generalized transformation. Readers can refer to [2].

In this paper, we focus on a class of cascade systems, where we cannot use the standard adaptive observer technique, either because the geometric transformation of the system is complex or because the parameters cannot be written on the affine form. We propose to derive a version of the adaptive observer-based approach, coupled with a linear parameter estimation method, for a class of nonlinear systems where the state vector $x$ in (1) can be partitioned into two components $x_{1}$ and $x_{2}$, such that (1) can be written in a cascade form:

$$
\left\{\begin{array}{l}
\dot{x_{1}}=\phi\left(x_{1}, u, t\right)+\psi\left(x_{1}, u, t\right) \theta \\
\dot{x_{2}}=\gamma\left(x_{2}, u, t\right)+\lambda(u, t) w \\
y=h\left(x_{2}\right)
\end{array}\right.
$$

where $w=C x_{1}$ is typically a linear partial measurement of $x_{1}$. The unknowns are $w, \theta, x_{1}$ and $x_{2}$ as shown in Figure 1.

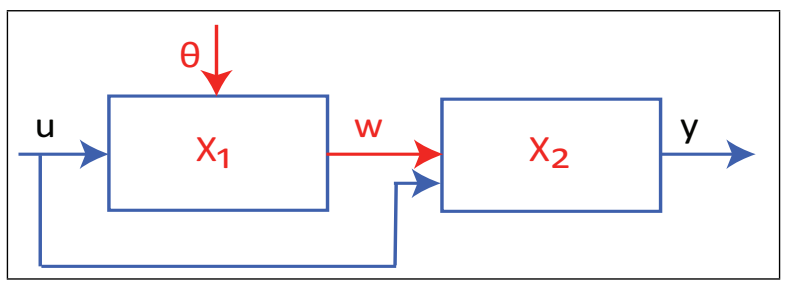

Fig. 1. Studied cascade system

For systems illustrated in Figure 1 and which can be put on the form (3), the main idea is to consider the underlying sub-systems separately in a hierarchical way. In other words, if the second subsystem is the observed one:

$$
\left\{\begin{array}{l}
\dot{x_{2}}=\gamma\left(x_{2}, u, t\right)+\lambda(u, t) w \\
y=h\left(x_{2}\right)
\end{array}\right.
$$

then we can apply an adaptive observer for state affine systems method to estimate both the state $x_{2}$ and the unknown input $w$. In a second step, we consider the first subsystem:

$$
\left\{\begin{array}{l}
\dot{x_{1}}=\phi\left(x_{1}, u, t\right)+\psi\left(x_{1}, u, t\right) \theta \\
w=C x_{1}
\end{array}\right.
$$

for which we propose to apply the modulating functions method to estimate the parameters in the linear case. Note that, due to the application that we will present in the next section, we focus only on the parameters estimation, but the method can be used in the general case of state and parameter estimation. 


\section{Application to THE BALLOON MODEL}

The nonlinear Balloon model can be represented in a statespace form:

$$
\begin{aligned}
& \left\{\begin{array}{l}
\dot{f}=s \\
\dot{s}=-\kappa_{f}(f-1)-\kappa_{s} s+\epsilon u(t) \\
\dot{v}=\frac{1}{\tau}\left(f-v^{\frac{1}{\alpha}}\right) \\
\dot{q}=\frac{1}{\tau}\left(f \frac{1-\left(1-E_{0}\right)^{\frac{1}{f}}}{E_{0}}-v^{\frac{1}{\alpha}-1} q\right)
\end{array}\right. \\
& y=V_{0}\left(k_{1}(1-q)+k_{2}\left(1-\frac{q}{v}\right)+k_{3}(1-v)\right)
\end{aligned}
$$

where the state vector $x$ is a set of non measurable variables which are:

- the blood inflow $f$,

- the flow-inducing signal $s$,

- the blood venous volume $v$,

- the veins deoxyhemoglobin content $q$,

$u$ is the input of the model which represents the stimulus function, and $y$ is the output given by the BOLD signal.

The parameters of the model can be split into an unknown part which includes the neural efficiency $\epsilon$, the flow decay $\kappa_{s}$, the flow time constant $\kappa_{f}$, and a known set, mostly invariant from subject to subject and from region to region of the brain. This set comprises the venous transit time $\tau$, Grub's parameter $\alpha$, the oxygen extraction at rest $E_{0}$ and the blood volume fraction at rest $V_{0}$.

To apply the estimation method presented above, we take: $x_{1}=[f, s]^{T}, x_{2}=[v, q]^{T}, \theta=\left[\kappa_{s}, \kappa_{f}, \epsilon\right]^{T}$ and $C=[1,0]$.

\section{A. Adaptive observer for unknown input and state estimation}

In this section, we focus on the estimation of state and input for the system:

$$
\begin{gathered}
\dot{x}=G(x, f)=\left\{\begin{array}{l}
\frac{1}{\tau}\left(f-v^{\frac{1}{\alpha}}\right) \\
\frac{1}{\tau}\left(f \frac{1-\left(1-E_{0}\right)^{\frac{1}{f}}}{E_{0}}-v^{\frac{1}{\alpha}-1} q\right)
\end{array}\right. \\
y=H(x)=V_{0}\left(k_{1}(1-q)+k_{2}\left(1-\frac{q}{v}\right)+k_{3}(1-v)\right)
\end{gathered}
$$

where $x=[v, q]^{T}$.

For this special case, the variables are normalized to the rest state and variations are small around the equilibrium corresponding to absence of neural activity $(u \equiv 0)$.

We apply an adaptive observer to the linearized system around the rest state corresponding to $x_{0}=[1,1]^{T}$ and $f_{0}=$ 1 , and show the asymptotic convergence of the estimated state to the real one, taking into account the physics of the system (range of variation of the main variables).

1) The observer structure: Let $z=x-x_{0}, w=f-f_{0}$ and $\zeta=y-C x_{0}$ be respectively the state, the input and the measurement of system (5) around the equilibrium point. It can now be written in the form:

$$
\left\{\begin{array}{l}
\dot{z}=A z+B w+\xi(z) \\
\zeta=C z
\end{array}\right.
$$

where

$$
A=\left.\frac{\partial G(x, f)}{\partial x}\right|_{x_{0}}=\frac{1}{\tau}\left(\begin{array}{cc}
-1 / \alpha & 0 \\
1-1 / \alpha & -1
\end{array}\right)
$$

$$
\begin{gathered}
B=\left.\frac{\partial G(x, f)}{\partial f}\right|_{f_{0}}=\frac{1}{\tau}\left[\begin{array}{ll}
1 & 1+\frac{1-E_{0}}{E_{0}} \ln \left(1-E_{0}\right)
\end{array}\right]^{T}, \\
C=\left.\frac{\partial H(x)}{\partial x}\right|_{x_{0}}=V_{0}\left[\begin{array}{l}
\left.\left(-k_{3}+k_{2}\right) \quad\left(-k_{1}-k_{2}\right)\right] .
\end{array}\right.
\end{gathered}
$$

and $\xi(z)$ is the modeling error. It is worth-noting that since the state vector is bounded by definition the non linear term can be bounded by:

$$
\|\xi(z)\| \leq \delta
$$

The state and input estimation is addressed using the adaptive observer proposed in [25]. Such observers have been developed in the linear case. In our case, we apply the observer to system (7). Thus, we get:

$$
\left\{\begin{array}{l}
\dot{\hat{z}}=A \hat{z}+B \hat{w}+(L+h K)(\zeta-\hat{\zeta}) \\
\dot{\hat{w}}=K(\zeta-\hat{\zeta}) \\
\dot{h}=(A-L C) h+B \\
\hat{\zeta}=C \hat{z}
\end{array}\right.
$$

with $K=\mu h^{T} C^{T}, \mu>0$ and $L$ chosen such that $A-L C$ is a Hurwitz matrix.

2) The convergence analysis:

Proposition 1. Consider the hemodynamic model described by equations (5) and (6) and its linearized version given by equation (7) with the assumption (8). Let us consider the adaptive observer in (9) such that the following conditions satisfy:

$\left(C_{1}\right): A-L C$ is a Hurwitz matrix,

$\left(C_{2}\right)$ : there exist two positive matrices $P$ and $Q$ satisfying the following equation:

$$
(A-L C)^{T} P+P(A-L C)=-Q,
$$

$\left(C_{3}\right)$ : the lowest eigenvalue of $Q$ and the highest eigenvalue of $P$, denoted respectively by $\lambda_{\min }(Q)$ and $\lambda_{\max }(P)$, satisfy the following relation:

$$
\frac{\lambda_{\min }(Q)}{\lambda_{\max }(P)}>\frac{2 \delta}{M} \text {. }
$$

where $M$ is a given large number.

Then, the observation errors $z-\hat{z}$ and $w-\hat{w}$ converge asymptotically to zero.

Proof: Let us denote the estimation errors of the state and the input respectively by $e_{z}:=z-\hat{z}$ and $e_{w}:=w-\hat{w}$. Then, using (7) and (9) we get:

$$
\begin{aligned}
\dot{e}_{z} & =(A-L C) e_{z}+\xi(z)+B e_{w}-h \dot{\hat{w}} . \\
\dot{e}_{w} & =-K C e_{z} .
\end{aligned}
$$

Let us denote $\bar{e}$ the modified error as follows:

$$
\bar{e}=e_{z}-h e_{w} .
$$

Then, using (12) we get:

$$
\begin{aligned}
\dot{\bar{e}}=(A & -L C)\left(\bar{e}+h e_{w}\right)+\xi(z)+B e_{w} \\
& -h \dot{\hat{w}}-h \dot{e}_{w}-\dot{h} e_{w} .
\end{aligned}
$$


Assume that, the neural activity estimate dynamics is faster than the real neural activity dynamics, i.e. we assume:

$$
\dot{e}_{w} \simeq-\dot{\hat{w}}
$$

Therefore, using (16) in (15) it yields:

$$
\dot{\bar{e}} \simeq(A-L C) \bar{e}+\xi(z)+((A-L C) h+B) e_{w}-\dot{h} e_{w} .
$$

By using the relation $\dot{h}=(A-L C) h+B$ given in (9), we obtain:

$$
\dot{\bar{e}} \simeq(A-L C) \bar{e}+\xi(z) .
$$

Let us take the following Lyapunov function:

$$
V=\bar{e}^{T} P \bar{e} .
$$

Then, the first order derivative with time of $V$ is given by:

$$
\dot{V}=\dot{\bar{e}}^{T} P \bar{e}+\bar{e}^{T} P \dot{\bar{e}}
$$

Hence, using (17), one has:

$$
\begin{gathered}
\dot{V}=\bar{e}^{T}\left[(A-L C)^{T} P+P(A-L C)\right] \bar{e} \\
+\xi^{T}(z) P \bar{e}+\bar{e}^{T} P \xi(z) .
\end{gathered}
$$

If the conditions $\left(C_{1}\right)$ and $\left(C_{2}\right)$ are satisfied, then we have:

$$
\dot{V}=-\bar{e}^{T} Q \bar{e}+\xi^{T}(z) P \bar{e}+\bar{e}^{T} P \xi(z)
$$

According to (8), the nonlinear term $\xi$ is bounded. Moreover, since $P$ and $Q$ are positive matrices, one can deduce the following inequality:

$$
\dot{V} \leq\left[-\lambda_{\min }(Q)\|\bar{e}\|+2 \delta \lambda_{\max }(P)\right]\|\bar{e}\|
$$

For $\|\bar{e}\| \leq M$ and the condition $\left(C_{3}\right)$ satisfied, we can conclude that $\dot{V}$ is negative definite. Consequently, $\dot{V}$ is asymptotically stable, i.e. we have:

$$
\bar{e} \longrightarrow 0
$$

Using (13) and (14), we get:

$$
\dot{e}_{w}=-K C\left(\bar{e}+h e_{w}\right)
$$

Note that the dynamic of $\bar{e}$ is independent of $w$, and $\bar{e}$ is bounded and tends to zero when $t \rightarrow \infty$. Further, if we take $K=\mu h^{T} C^{T}$ with $\mu>0$, then the following autonomous system

$$
\dot{e}=-K C h e
$$

is exponentially stable. Consequently, by using the same arguments as done in [25], [13] we can deduce that:

$$
e_{w} \longrightarrow 0 \text { when } t \longrightarrow \infty \text {. }
$$

\section{B. Application of modulating functions method}

1) Modulating functions method: Let $l \in \mathbb{N}^{*}, T \in \mathbb{R}_{+}^{*}$, and $g$ be a function satisfying the following properties:

$\left(P_{1}\right): g \in \mathcal{C}^{l}([0, T])$;

$\left(P_{2}\right): g^{(j)}(0)=g^{(j)}(T)=0, \forall j=0,1, \ldots, l-1$, then $g$ is called $l^{\text {th }}$ order modulating function on [0,T] [19].

By using modulating functions, one can estimate directly parameters of the following differential equation:

$$
y(t)+\sum_{i=1}^{n} a_{i} y^{(i)}(t)=\sum_{i=0}^{m} b_{i} u^{(i)}(t), n \geq m,
$$

where $y$ is the output, $u$ is the input, and $a_{i}, b_{i}$ are the unknown parameters. The main idea of the modulating functions method is as follows: let $g$ be $l^{\text {th }}$ order modulating function on $[0, \mathrm{~T}]$ with $l=\max (n, m)$, then we multiply both sides of (18) by $g$, and integrate from 0 to $T$. By applying integration by parts and using the properties $\left(P_{1}\right)$ and $\left(P_{2}\right)$, we can obtain the following algebraic integral equation:

$$
\begin{aligned}
\sum_{i=0}^{n}(-1)^{i} a_{i} \int_{0}^{T} g^{(i)}(\tau) y(\tau) d \tau= & \\
& \sum_{i=0}^{m}(-1)^{i} b_{i} \int_{0}^{T} g^{(i)}(\tau) u(\tau) d \tau
\end{aligned}
$$

where $a_{0}=1$. In order to calculate all parameters $a_{i}, b_{i}$, we need to generate at least $n+m+1$ linearly independent algebraic integral equations similar to (19). The proposed approach gives explicit formulae for the parameters, which gains good robustness against corrupting noises from the integrals [5], [15]. Moreover, we do not need to estimate the derivatives of input-output signals.

2) Parameters estimation: Let us consider the following linear system:

$$
\left\{\begin{array}{l}
\dot{f}=s \\
\dot{s}=-\kappa_{f}(f-1)-\kappa_{s} s+\epsilon u \\
\hat{f}=f+\varpi
\end{array}\right.
$$

where $\hat{f}$ is an estimation of $f$, and $\varpi$ is the corresponding estimation error. In the following proposition, we are going to estimate the parameter vector $\theta=\left[\kappa_{f}, \kappa_{s}, \epsilon\right]^{T}$ using the modulating functions method, the input $u$ and $\hat{f}$.

Proposition 2. Let $g_{i}$, for $i=1, \cdots, M$, be a set of second order modulating functions on $\left[0, T_{i}\right]$, where $0<T_{i}<\cdots<$ $T_{M}$. Then, the parameter vector $\theta=\left[\kappa_{f}, \kappa_{s}, \epsilon\right]^{T}$ can be estimated by solving the following linear system:

$$
\hat{D} \hat{\theta}=\hat{b}
$$

where, for $i=1, \cdots, M, \hat{D}(i, 1)=-\int_{0}^{T_{i}} g_{i}(t) \hat{f}(t) d t$, $\hat{D}(i, 2)=\int_{0}^{T_{i}} \dot{g}_{i}(t) \hat{f}(t) d t, \hat{D}(i, 3)=\int_{0}^{T_{i}} g_{i}(t) u(t) d t$, and $\hat{b}(i)=\int_{0}^{T_{i}} \ddot{g}_{i}(t) \hat{f}(t) d t$.

Proof: According to (20), we can obtain the following differential equation:

$$
\ddot{f}(t)=-\kappa_{f}(f(t)-1)-\kappa_{s} \dot{f}(t)+\epsilon u(t) .
$$


By multiplying both sides of (21) by $g_{i}$, and by integrating from 0 to $T_{i}$, we get:

$$
\begin{aligned}
\int_{0}^{T_{i}} g_{i}(t) \ddot{f}(t) d t=-\kappa_{f} \int_{0}^{T_{i}} g_{i}(t)(f(t)-1) d t \\
-\kappa_{s} \int_{0}^{T_{i}} g_{i}(t) \dot{f}(t) d t+\epsilon \int_{0}^{T_{i}} g_{i}(t) u(t) d t .
\end{aligned}
$$

Then, by applying integration by parts and using the properties $\left(P_{1}\right)-\left(P_{2}\right)$, we obtain:

$$
\begin{aligned}
\int_{0}^{T_{i}} \ddot{g}_{i}(t) & f(t) d t=-\kappa_{f} \int_{0}^{T_{i}} g_{i}(t) f(t) d t \\
& +\kappa_{s} \int_{0}^{T_{i}} \dot{g}_{i}(t) f(t) d t+\epsilon \int_{0}^{T_{i}} g_{i}(t) u(t) d t .
\end{aligned}
$$

Hence, we obtain the following linear system:

$$
D \theta=b,
$$

where, for $i=1, \cdots, M, D(i, 1)=-\int_{0}^{T_{i}} g_{i}(t) f(t) d t$, $D(i, 2)=\int_{0}^{T_{i}} \dot{g}_{i}(t) f(t) d t, D(i, 3)=\int_{0}^{T_{i}} g_{i}(t) u(t) d t$, and $b(i)=\int_{0}^{T_{i}} \ddot{g}_{i}(t) f(t) d t$. Consequently, the parameter $\theta$ can be estimated by solving (22) and using $\hat{f}$.

\section{NUMERICAL RESUlTS}

To illustrate the method presented above, a set of synthetic data was generated using the Balloon model, with the parameters values summarized in Table I. The adaptive observer, described in the previous section, is then applied to the continuous system using a forth order Runge Kutta scheme for integration. The time step for integration is $T_{e}=0.1 s$, and a white gaussian noise, corresponding to a Signal to Noise Ratio (SNR) of 30 is added to the simulated BOLD signal as shown in Figure 2. The estimation of blood flow is illustrated in Figure 3, blood volume and deoxyhemoglobin content are shown in Figure 4. In order to estimate the parameter $\theta=\left[\kappa_{f}, \kappa_{s}, \epsilon\right]^{T}$ using Proposition 2 , we use a sliding integration window of finite length in our identification procedure with the following modulating functions: for $i=1, \cdots, 6$,

$$
g_{i}(t)=\left(T_{i}-t\right)^{3} t^{3},
$$

where $T_{i}=5+2 i$. Consequently, the length of the sliding integration window is equal to $T_{6}=17$, and our identification procedure begins at $17 \mathrm{~s}$. Moreover, we apply the numerical trapezoidal integration method to approximate each integration window. Let us remark that, due to the filtering property of the observer, the estimation may present a time delay, as shown in Figure 3. Nevertheless, in practical situations, the value of the delay can easily be estimated. Indeed, the linear subsystem presents no modeling delays, allowing to provide an estimate of the observer delay as the difference between the triggering times of $u$ and $\hat{f}$. Therefore, to avoid errors in the parameters' estimation, we shift the input $u$ backward with a value equal to the time-delay. In this example, we take $8 T_{e}$ as the value of the time-delay. The study of the value of the time-delay is out of the scope of this paper. Finally, we obtain the estimated parameters in Figure 5,

and the corresponding relative estimation errors in Figure 6. Let us mention that since the modulating functions method uses a sliding integration window of finite length, it is a non-asymptotic method. The convergence of the estimations depends on the time step $T_{e}$. Hence, we can improve the estimation errors in Figure 6 by reducing $T_{e}$ (see [5], [15] for more details).

\begin{tabular}{|c|c|}
\hline Parameters & Values \\
\hline$\epsilon$ & 0.38 \\
\hline$\kappa_{s}$ & 1.25 \\
\hline$\kappa_{f}$ & 2.5 \\
\hline$\tau$ & 1 \\
\hline$\alpha$ & 0.2 \\
\hline$E_{0}$ & 0.8 \\
\hline
\end{tabular}

TABLE I

PARAMETERS OF THE BALLOON MODEL

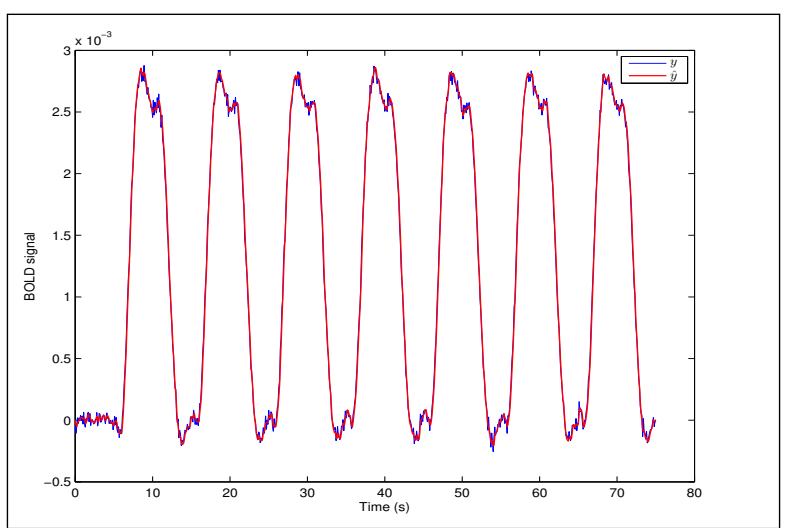

Fig. 2. True noisy (blue) and estimated (red) BOLD signals

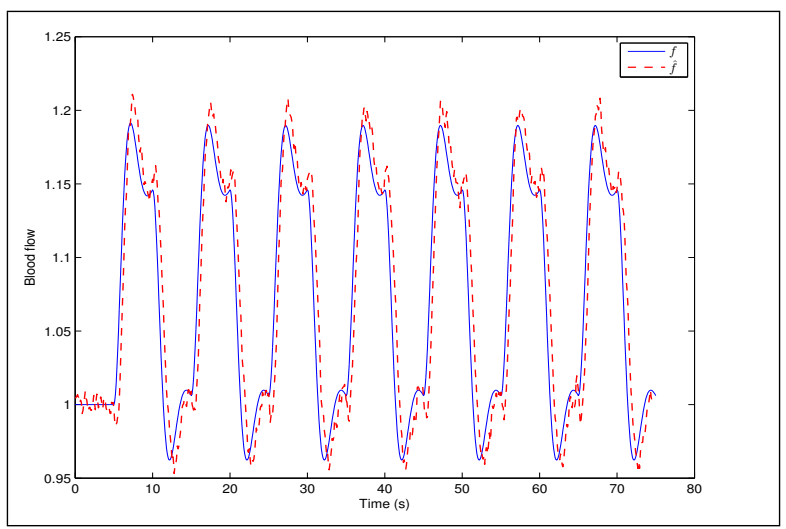

Fig. 3. True unknown (continuous line) and estimated (dashed line) blood flow $f$

\section{CONCLUSIONS}

The joint estimation of states and parameters for a nonlinear hemodynamic model has been addressed in this work. The studied model cannot be presented as a state affine system, but falls in a class of cascade systems where it is possible to couple an adaptive observer with a parameter 


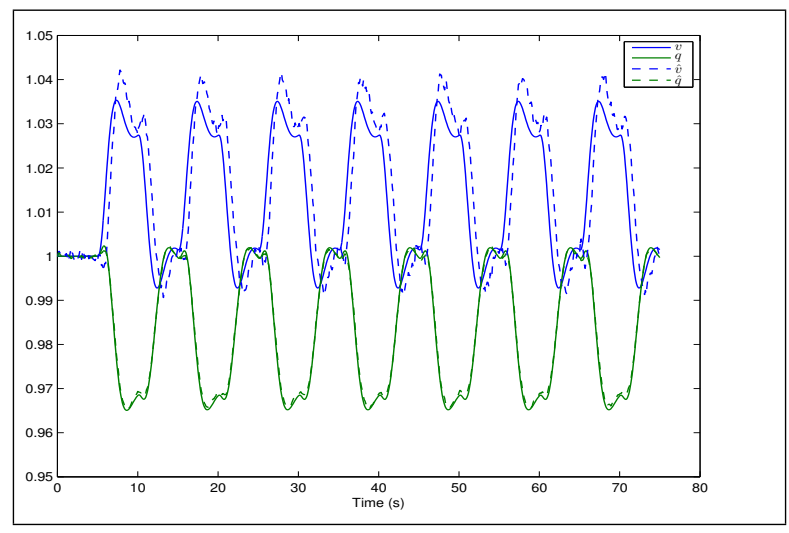

Fig. 4. Blood volume $v$ (blue) and deoxyhemoglobin content $q$ (green). The reference unknown signals are plotted in continuous lines while the estimated ones are in dashed lines

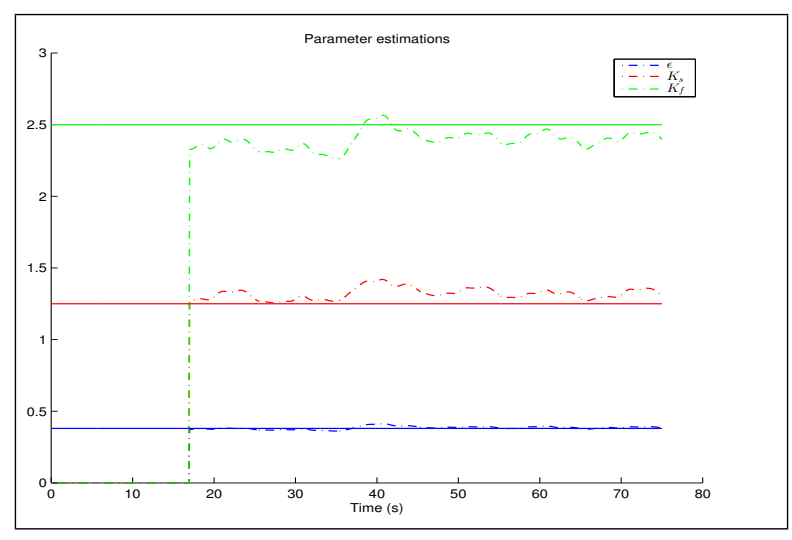

Fig. 5. True parameters and their estimations

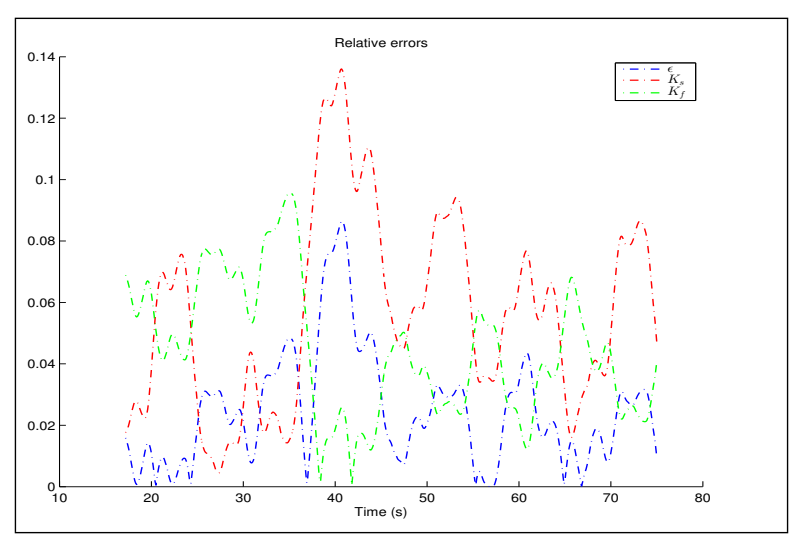

Fig. 6. Relative parameter estimation errors

identification technique. The adaptive observer has shown robustness against bounded model nonlinearities. Parameter estimation was performed using the algebraic modulating function technique, which provides accurate estimates avoiding the computation of noisy signals derivatives. The proposed method has been validated on a set of synthetic data and has shown satisfying results. Future work will address the validation of the approach on real data sets and emphasize the joint estimation of states, parameters and the input (the neural activation) of the Balloon model.

\section{REFERENCES}

[1] G. Bastin and M.R. Gevers, Stable adaptive observers for nonlinear time-varying systems, IEEE Transactions on Automatic Control, vol. 33, 1988, pp. 650-658.

[2] G. Besancon, Remarks on nonlinear adaptive observer design, Systems and Control Letters, vol. 41, 2000, pp. 271-280.

[3] R.B. Buxton, E.C. Wrong and L.R. Frank, Dynamics of Blood flow and Oxygenation Changes during Brain activation: The Balloon Model, Magnetic Resonance in Medicine, vol. 39, 1998, pp. 855-864.

[4] T. Deneux and O. Faugeras, Using nonlinear models in fMRI data analysis: model selection and activation detection, Neuroimage, vol. 32, pp. 1669-1689.

[5] M. Fliess, Analyse non standard du bruit, C.R. Acad. Sci. Paris Ser. I, 342, 2006, pp. 797-802.

[6] K.J. Friston, A. Michelli, R. Turner and C.J. Price, Nonlinear responses in fMRI: The Balloon Model, Volterra Kernels and other Hemodynamics, Neuroimage, vol. 12, 2000, pp. 466-477.

[7] K.J. Friston, N. Trujillo-Barreto and J. Daunizeau, DEM: a variational treatment of dynamic systems, Neuroimage, vol. 41, 2008, pp. 849885.

[8] H. Garnier, L. Wang and Peter C. Young, Direct Identification of Continuous-time Models from Sampled Data: Issues, Basic Solutions and Relevance, Garnier, Hugues; Wang, Liuping (Eds.), Identification of continuous-time models from sampled data, Springer-Verlag, London, 2008, pp. 1-29.

[9] Q.P. Ha and H. Trinh, State and input simultaneous estimation for a class of nonlinear systems, Automatica, vol. 40, no. 10, 2004, pp. 1779-1785.

[10] M. Havlicek and K.J. Friston, J. Jan, M. Brazdil and V. D. Calhoun, Dynamic Modeling of neural responses in fMRI using cubature Kalman filtering, Neuroimage, vol. 56, 2011, pp.2109-2128.

[11] G. Kreisselmeier, Adaptive observers with exponential rate of convergence, IEEE Transactions on Automatic Control, vol. 22, 1977 , pp.2-8.

[12] A.J. Krener and A. Isidori, Linearization by output injection and nonlinear observers, Systems Control Letters, vol. 3, 1983, pp. 4452

[13] T.M. Laleg-Kirati, H. Arabi, M. Tadjine and C. Zayane, Estimation of the Neuronal activation using fMRI data: an observer-based approach, the 2013 American Control Conference, 2013, Washington, DC, USA.

[14] D.Y. Liu, T.M. Laleg-Kirati, O. Gibaru and W. Perruquetti, Identification of fractional order systems using modulating functions method, the 2013 American Control Conference, 2013, Washington, DC, USA

[15] D.Y. Liu, O. Gibaru and W. Perruquetti, Parameters estimation of a noisy sinusoidal signal with time-varying amplitude, 19th Mediterranean conference on Control and automation (MED'11), 2011, Corfu, Greece.

[16] L. Ljung, Asymptotic behavior of the extended Kalman filter as a parameter estimator for linear systems, IEEE Transactions on Automatic Control, vol. 24, no. 1, 1979, pp. 36-50.

[17] R. Marino and P. Tomei, Adaptive observers with arbitrary exponential rate of convergence for nonlinear systems, IEEE Transactions on Automatic Control, vol. 40, no. 7, 1995, pp. 1300-1304.

[18] R. Marino and P. Tomei, Global adaptive output-feedback control of nonlinear systems. I. Linear parameterization, IEEE Transactions on Automatic Control, vol. 38, no. 1, 1993, pp. 17-32.

[19] H.A. Preising and D.W.T. Rippin, Theory and application of the modulating function method I: Review and theory of the method and theory of the spline type modulating functions, Computers and Chemical Engineering, vol. 17, 1993, pp. 1-16.

[20] K.C. Veluvolu and Y.C. Soh, High-Gain Observers With Sliding Mode for State and Unknown Input Estimations, IEEE Transactions on Industrial Electronics, vol. 56, no. 9, 2009, pp.3386-3393.

[21] G.P. Rao and H. Unbehauen, Identification of continuous-time systems, IEE Proc. Control Theory Appl., vol. 153, no. 2, 2006, pp. 185-220.

[22] J.J. Riera, J. Watanabe, I. Kazuki, M. Naoki, E. Aubert, T. Ozaki and R. Kawashima, A state-space model of the hemodynamic approach: nonlinear filtering of BOLD signals, Neurolmage, vol. 21, 2004, pp. $547-567$.

[23] M. Shinbrot, On the analysis of linear and nonlinear systems, Transactions on ASME, vol. 79, 1957, pp. 547-552.

[24] Q. Zhang, Adaptive observer for multi input multi output linear time varying systems, IEEE Transactions on Automatic Control, vol. 3 , 2002, pp. 525-529. 